\title{
Evaluation of hyaluronic acid-P40 conjugated cream in a mouse model of dermatitis induced by oxazolone
}

\author{
KATIA MANGANO $^{1}$, FRANCA VERGALITO ${ }^{2}$, SANTA MAMMANA ${ }^{1}$, ANDREA MARIANO $^{2}$, \\ ROCCO DE PASQUALE ${ }^{3}$, ANTONIA MELOSCIA ${ }^{2}$, SILVIA BARTOLLINO ${ }^{2}$, \\ GERMANO GUERRA $^{2}$, FERDINANDO NICOLETTI ${ }^{1}$ and ROBERTO DI MARCO ${ }^{2}$ \\ ${ }^{1}$ Department of Biomedical and Biotechnological Sciences, University of Catania, I-95124 Catania; \\ ${ }^{2}$ Department of Medicine and Health Sciences, University of Molise, I-86100 Campobasso; \\ ${ }^{3}$ Department of Dermatology, Policlinico-Vittorio Emanuele Hospital, I-95124 Catania, Italy
}

Received July 15, 2016; Accepted January 6, 2017

DOI: $10.3892 /$ etm.2017.4810

\begin{abstract}
P40 is a particulate fraction or fragment isolated from Corynebacterium granulosum, which exhibits a wide spectrum of pharmacological functions including antitumor, antibacterial, phagocytic, antiviral and cytokine induction effects. In the present study, the immunomodulatory potential of $\mathrm{P} 40$-conjugated with hyaluronic acid was assessed in a mouse model of dermatitis induced by oxazolone. Oxazolone-induced allergic contact dermatitis is a T cell-mediated Th2-like hypersensitivity reaction, which mimics the corresponding reaction in humans. Female cluster of differentiation-1 mice were sensitized on days 0 and 1 by the application of $2 \%$ oxazolone onto a shaved back. The disease was induced by re-challenge on day 7 using 15\% oxazolone in the inner and outer of the left ears of the mice. Mice were topically treated with hyaluronic acid-P40 conjugate cream or with placebo to the inner and outer surface of the left ear for 7 consecutive days starting from $1 \mathrm{~h}$ after the sensitization. A significant reduction in ear thickness and weight and in edema and leukocyte recruitment were observed in the mice treated with hyaluronic-P40 conjugate cream compared with mice treated with the cream base alone $(\mathrm{P}<0.05)$. Thus, $\mathrm{P} 40$-conjugated with hyaluronic acid may constitute an innovative dermatitis treatment.
\end{abstract}

\section{Introduction}

P40 is a particulate fraction or fragment isolated from Corynebacterium granulosum (now called Propionibacterium acnes), that was first described in 1970 by a group of researchers at the Pasteur Institute in Paris, and has previously been studied for its pleiotropic effect (1). P40 has been demonstrated to perform

Correspondence to: Professor Roberto Di Marco, Department of Medicine and Health Sciences, University of Molise, 1 Via Francesco De Sanctis, I-86100 Campobasso, Italy

E-mail: roberto.dimarco@unimol.it

Key words: dermatitis, oxazolone, immunomodulation, $\mathrm{P} 40$ fraction a wide spectrum of pharmacological functions including, antitumor effects via a marked inhibitory activity towards 'liquid' and solid tumors in mice (2), adjuvant effects (3), immunomodulatory effects $(4)$, effects on phagocytosis $(5,6)$, antimicrobial properties on bacterial infections (7), effects on viral infections (8), cytokine induction (9) and effects on Escherichia coli infections (10). In animal models, it has been demonstrated that $\mathrm{P} 40$ exhibits dichotomous action in immune-mediated diseases by increasing the production of antibodies against sheep red blood cells and augmenting the resistance of mice towards bacterial infections (4). P40 may also enhance the primary and secondary immune responses to a stimulus involving a particulate antigen, and under defined conditions, overcome the immunosuppressive effects of cyclophosphamide $(4,11)$.

Furthermore, a number of clinical trials have been performed with a fraction of $\mathrm{P} 40$ to assess its involvement in recurrent respiratory system $(12,13)$ and genitourinary tract infections (14), allergies (15) and breast cancer (16,17). Specifically, it was observed in recurrent respiratory infection that P40 treatment induced a significant reduction in infection rate and the duration of antibiotic therapy required (12). Similarly, in recurrent urogenital infection, P40 treatment induced a reduction in infection rate (14), while in the treatment of allergies, a Corynebacterium granulosum-derived P40-ovalbumin conjugate was demonstrated to significantly inhibit mast cell degranulation (15). It also proved to be capable of protecting against lethal systemic anaphylactic shock in sensitized mice (15).

Due to the established properties of P40, the present study investigated its potential immunomodulant effects when conjugated with hyaluronic acid $(\mathrm{Ha})$ in a mouse model of dermatitis induced by oxazolone. $\mathrm{P} 40$ was conjugated with $\mathrm{Ha}$ as it is a not immunogenic compound and it has a molecular weight (MW) of $500 \mathrm{kDa}$, which prevents the systemic absorption of P40 and, therefore, its degradation. The selection of this MW was based on a previous study by Esser et al (18), which demonstrated that the size of Ha serves an important role in the immune-modulatory effect and, in particular, a high MW $\mathrm{Ha}\left(<1 \times 10^{6} \mathrm{kDa}\right)$ is anti-angiogenic, anti-inflammatory and immunosuppressive. This allows the Ha-based cream to be 
useful in the treatment of mild-to-moderate atopic dermatitis, in addition to preserve barrier function in the maintenance phase of dermatitis management (18).

Allergic contact dermatitis (ACD) is a T cell (Tc)-mediated delayed type hypersensitivity reaction, which mimics the processes occurring in human ACD and may be induced in mice by repeated epicutaneous application of oxazolone (19). Contact hypersensitivity (CHS) is an immunological response against a small chemical hapten that, during the sensitization phase, is absorbed and processed by dermal dendritic and Langerhans cells; these migrate to the draining lymph node where they present the antigen to naive Tc, leading to the formation of antigen-specific memory Tc (18). Subsequent contact with the same chemical results in the activation of memory Tc and the recruitment of inflammatory cells to the site of allergen exposure (19). In ACD, the adaptive immune response is elicited primarily through the activation and expansion of cytotoxic cluster of differentiation (CD)8+ Tc1 or CD4+ T helper (Th) 1 cells and Tc17/Th17 cells in a multi-step process (19). In murine CHS the primary effector cells are cytotoxic Tc1 cells (18). This immune response is observed in the elicitation phase and manifests as skin inflammation, which is diagnosed as ACD (20) and the clinical symptoms include a local augmented thickness and weight increase of the skin area where the re-challenge has been applied. Due to an ease of application and measurement of responses, in addition to accessibility of sampling and draining lymph nodes the second challenge is typically applied onto one ear, while the contralateral is left unchallenged as a control $(18,20)$.

Oxazolone-induced dermatitis is characterized by severe mononuclear cell recruitment with in situ production of type 1 and type 2 cytokines. This characteristic inflammatory skin damage means that oxazolone-dermatitis is a useful in vivo tool for pathogenic studies to select potential therapeutics for the treatment of inflammatory responses associated with the skin, including those already identified for certain forms of bullous disorders, cutaneous vasculitis and psoriasis (21).

Therefore, the present study aimed to evaluate the effects of Ha-P40 conjugate cream, administered via a prophylactic regimen, on the progression of oxazolone-induced dermatitis in mice.

\section{Materials and methods}

Animals. A total of 38 female mice (6-7 weeks old, 22-25 g) were used (Harlan Laboratories, Udine, Italy). The animals were kept at the animal facility of the Department of Biomedical Sciences, Section of Pathology and Oncology (Catania, Italy), under standard laboratory conditions (non-specific pathogen free) with ad libitum access to food and water. Animals were housed within a limited access rodent facility under controlled microbial conditions that excluded murine pathogens and were kept in groups of $\leq 5$ mice within polycarbonate cages (Tecniplast, Buguggiate, Italy), according to Italian legislation (each mouse had $180 \mathrm{~cm}^{2}$ space with $\geq 12 \mathrm{~cm}$ height) (22). Automatically controlled environmental conditions were set to maintain a temperature of $20-24^{\circ} \mathrm{C}$ with a relative humidity of $30-70 \%, 10-30$ air changes/h and a 12-h natural light/dark cycle. All mice were allowed to adapt to their environment for one week prior to the commencement of the study. All study protocols were approved by the local ethical committee OPBA (Organismo Preposto al Benessere degli Animali, Catania, Italy) and the Ministry of Health (Roma, Italy). The welfare of all animals was in accordance with the Directive 2010/63/UE approved by the Italian D.L. no. 26 on March 2014 (22).

Induction of oxazolone-induced contact/atopic dermatitis. All 36 mice were sensitized on days 0 and 1 by the application of $50 \mu \mathrm{l}$ 2\% oxazolone (Chimica; Merck KGaA, Darmstadt, Germany) in ethanol on the shaved back. The disease was elicited by re-challenge on day 7 with $10 \mathrm{ml} 15 \%$ oxazolone in the inner and outer left ear. The right ear was treated with the vehicle (ethanol) alone. The mice were sacrificed by $20 \% \mathrm{CO}_{2}$ inhalation, using a flow rate of $1 \mathrm{l} / \mathrm{min} \mathrm{CO}_{2}$, for a total of $10 \mathrm{~min}$. Euthanasia was confirmed by checking for the absence of heartbeat. The right and left ears were excised $18 \mathrm{~h}$ following sensitization (23).

Scoring. Inflammation was expressed as the percentage increase in ear thickness and/or ear weight in the treated left ear vs. the vehicle-treated right ear. Ear thickness was measured with a digital caliper. Ear weight was measured using an electronic balance. The extent of inflammation was calculated as follows: Ear swelling $(\%)=(1-\mathrm{a} / \mathrm{b}) \times 100$, where $\mathrm{a}$ is thickness/weight of the right (untreated) ear and $\mathrm{b}$ is the thickness/weight of the left (treated) ear.

Treatment. Ha-P40 conjugate cream or base cream (Claride Pharma SA, Lugano, Switzerland), were administered by topical application of $10 \mu \mathrm{l}$ to the inner and outer surface of the left ear. The base cream was composed of hyaluronic acid supplemented with aqua, cetearyl ethylhexanoate, cyclopentasiloxane, glycerin, steareth-2, cera alba, steareth-21, cetearyl alcohol, stearic acid, phenoxyethanol, potassium sorbate, tocopheryl acetate, Argania spinosa kernel oil, disodium EDTA, Prunus amygdalus dulcis oil, Rosa moschata seed oil, tocopherol and benzyl alcohol. The Ha-P40 conjugate cream was composed of base cream supplemented with 5\% hyaluronic acid-P40 conjugate (Claride Pharma SA). Two groups of mice $(n=9)$ were treated with Ha-P40 conjugate or base cream under a prophylactic regimen for 7 consecutive days beginning $1 \mathrm{~h}$ after the first sensitization. An additional group of mice $(n=9)$ was treated with $0.5 \%$ dexamethasone ointment (Laboratory Farmacologico Milanese, Caronno Pertusella, Italy), as a positive control under the same experimental regimen. A group of mice with dermatitis, $(n=9)$ was left untreated, as a negative control. Two additional healthy mice (without dermatitis) that did not receive treatment, were used to define baseline values of ear thickness and body weight (SHAM group).

Histological analysis. Following sacrifice, the right and left ears of each mouse were fixed in $10 \%(\mathrm{w} / \mathrm{v})$ formalin and maintained at $4^{\circ} \mathrm{C}$ for 1 week prior to histological examination. Samples were embedded in paraffin wax and cut into 4- $\mu \mathrm{m}$ sections. The sections were mounted onto slides and stained with hematoxylin and eosin. Ear edema and leukocyte recruitment were determined at x40 and x400 magnification, respectively, using a light microscope. A score from 1 to 5 was assigned to each mouse according the progression of edema 


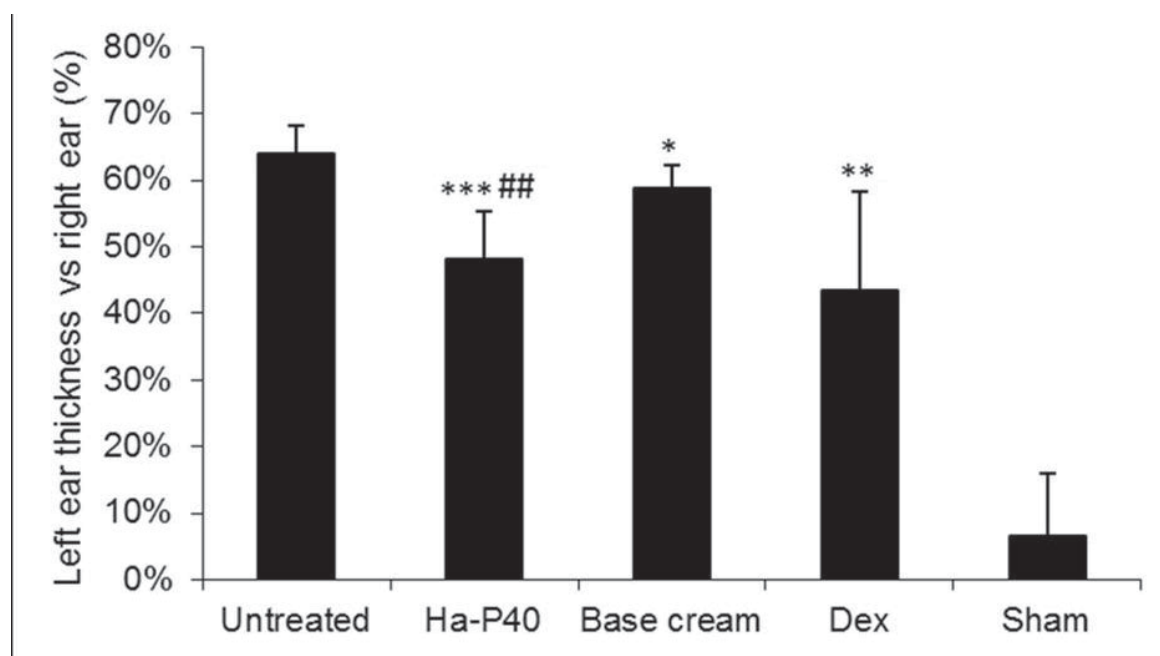

Figure 1. Effects of Ha-P40 conjugate cream on the treated, left ear thickness vs. the untreated right ear. Mice were topically treated in the inner and outer surface of the left ear with the conjugate cream or base cream or $0.5 \%$ Dex ointment for 7 consecutive days starting $24 \mathrm{~h}$ after the initial sensitization. Mice were sacrificed by $\mathrm{CO}_{2}$ inhalation $18 \mathrm{~h}$ following sensitization and the right and left ears were excised and thickness measured using a digital caliper. Data are presented as the mean \pm standard deviation. ${ }^{*} \mathrm{P}<0.05,{ }^{* * *} \mathrm{P}<0.01$ and ${ }^{* * *} \mathrm{P}<0.0001$ vs. untreated; ${ }^{\# \#} \mathrm{P}<0.01$ vs. base cream. Ha-P40, Hyaluronic acid-P40; Dex, Dexamethasone; Sham, healthy mice without dermatitis that did not receive any treatment.

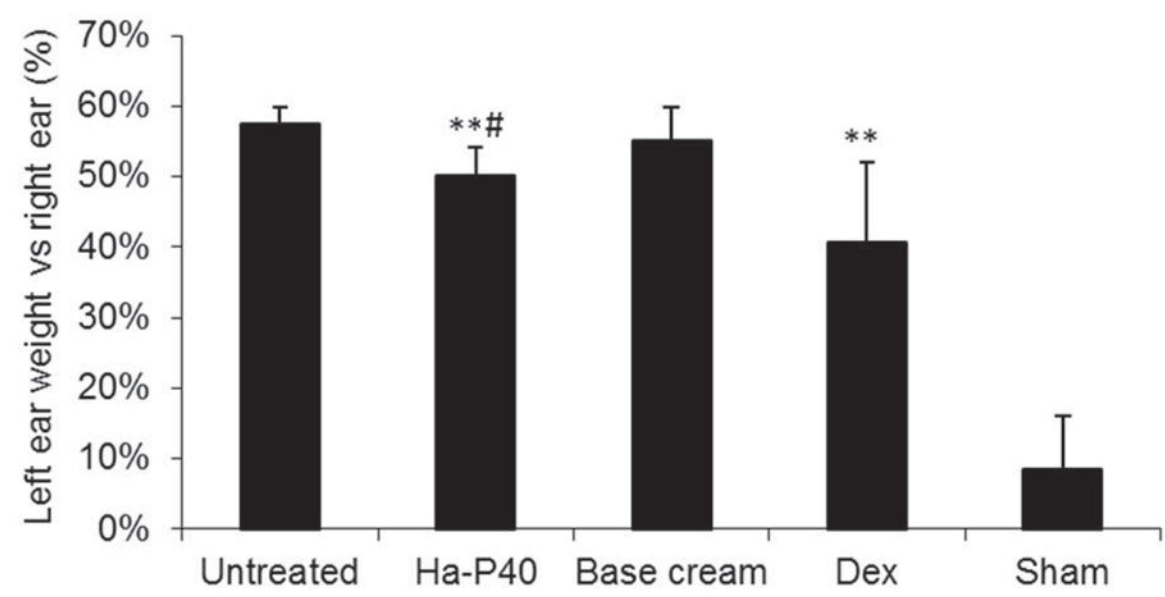

Figure 2. Effects of Ha-P40 conjugate cream on the treated, left ear weight vs. the untreated right ear. Mice were topically treated in the inner and outer surface of the left ear with the conjugate cream or base cream or $0.5 \%$ Dex ointment for 7 consecutive days starting $24 \mathrm{~h}$ after the initial sensitization. Mice were sacrificed by $\mathrm{CO}_{2}$ inhalation $18 \mathrm{~h}$ following sensitization and the right and left ears were excised and weighed. Data are presented as the mean \pm standard deviation. ${ }^{* *} \mathrm{P}<0.001$ vs. untreated; "P<0.05 vs. base cream. Ha-P40, Hyaluronic acid-P40; Dex, dexamethasone; Sham, healthy mice without dermatitis that did not receive any treatment.

and leukocyte number. The scoring system was as follows: 1 , No evident lesions; 2 , edema in the papillary dermis and subcutis without leucocyte infiltration; 3, edema in the papillary dermis and subcutis with mild leucocyte infiltration; 4, edema in the papillary dermis and subcutis with moderate leucocyte infiltration; 5 , edema in the papillary dermis and subcutis with severe leucocyte infiltration. An independent observer, blind to the drug treatments, provided the scores.

Statistical analysis. Results are presented as the mean percentage increase \pm standard deviation of left ear thickness and weight relative to that of the right ear. A Student's t-test was used to determine statistical significance and $\mathrm{P}<0.05$ was considered to represent a statistically significant difference. Sigma plot software version 12.0 (Systat software Inc, San Jose, CA, USA) was used to perform statistical analyses.

\section{Results}

Effects of Ha-P4O conjugate cream on ear thickness and weight. The oxazolone-induced dermatitis was successful: The untreated mice (mice with dermatitis left untreated as negative control group, $n=9$ ) exhibited a significant increase in ear thickness and weight compared with sham healthy mice (mice without dermatitis so to have reference values for ear weight and thickness and body weight, $\mathrm{n}=2$ ). Treatment with the positive control drug dexamethasone significantly reduced the weight and thickness of the left ear compared to the untreated mice (Figs. 1 and 2; $\mathrm{P}<0.01$. In addition, daily treatment for 7 consecutive days with base cream alone was able to significantly reduce left ear thickness relative to untreated mice (Fig. 1; P<0.05). Treatment with Ha-P40 conjugate cream enhanced the effects obtained with the 


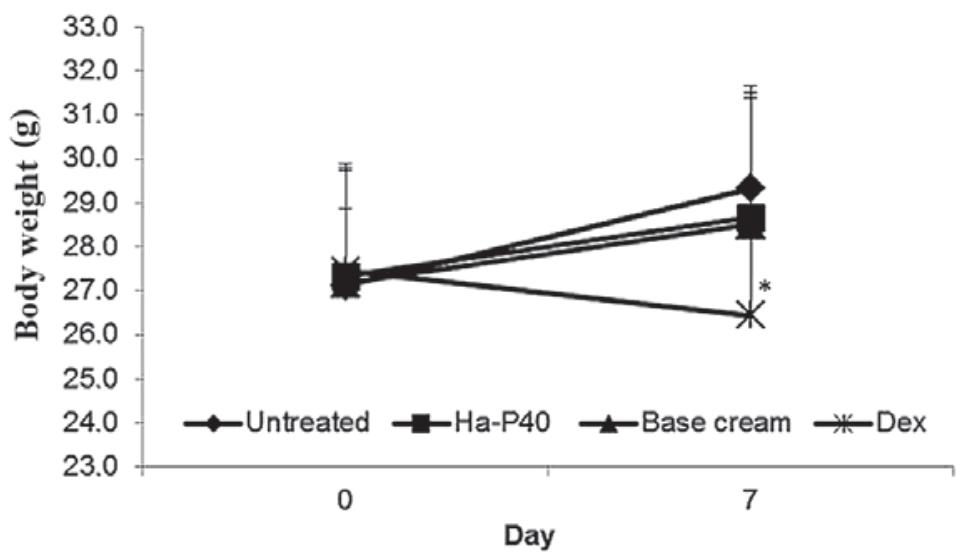

Figure 3. Effects of Ha-P40 conjugate cream on body weight over 7 days of treatment. Mice were weighed on day 0 of sensitization and day 7 of elicitation. Weight was assessed by Student's t-test. Data are presented as the mean \pm standard deviation. ${ }^{~} \mathrm{P}<0.05$ vs. untreated. Ha-P40, Hyaluronic acid-P40; Dex, dexamethasone.
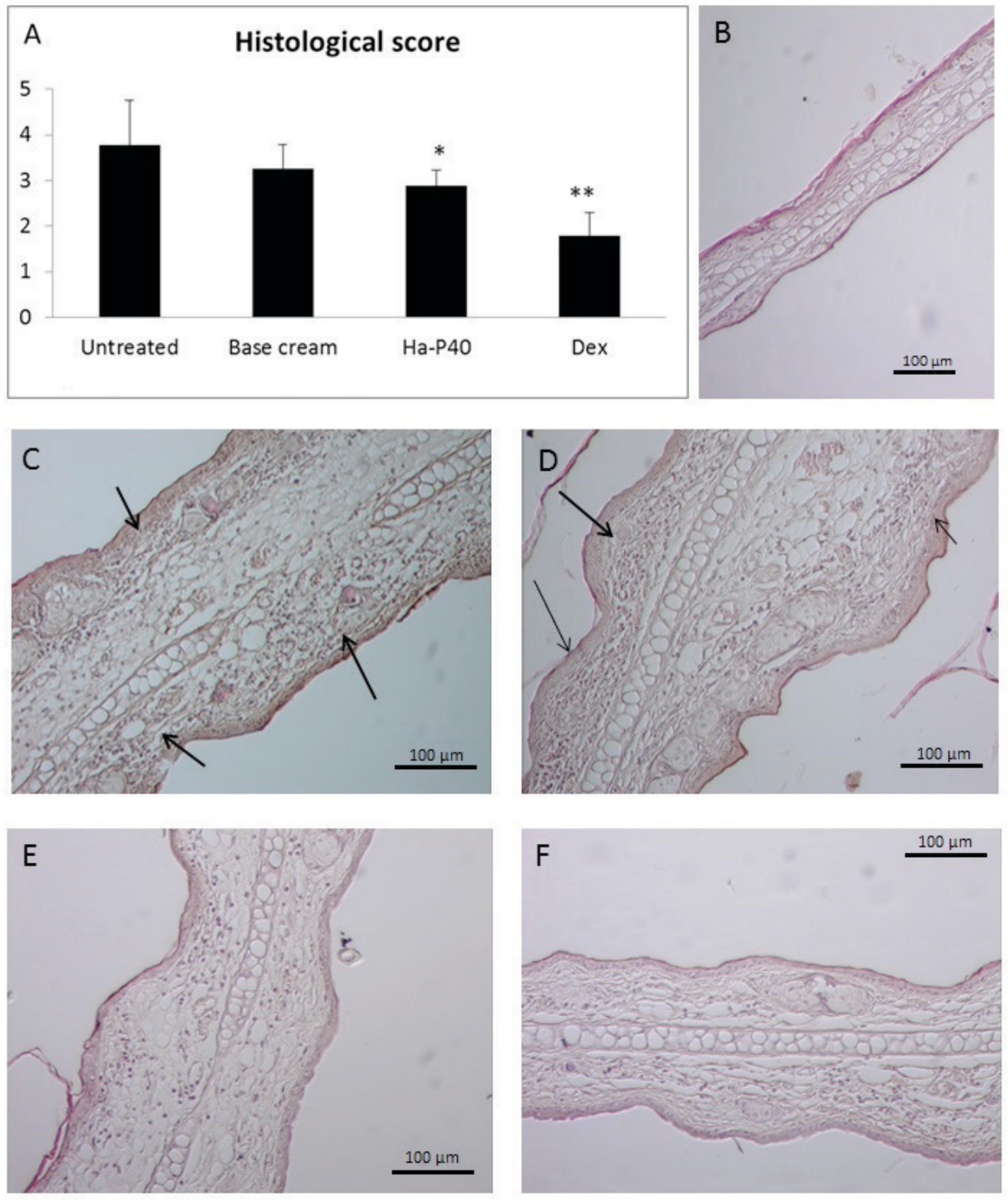

Figure 4. Effects of Ha-P40 conjugate cream on the histology. (A) Effects on histological score following treatment with Ha-P40 conjugate cream, as determined in the left ears of cluster of differentiat ion-1 mice. Score was determined by an experimenter blinded to treatment group. (B) Representative histological image stained with hematoxylin and eosin of ears from healthy mice prior to oxaxolone sensitization. Oxazolone-induced dermatitis in (C) untreated mice, (D) base cream-treated mice, (E) Ha-P40 conjugate-treated mice and (F) Dex-treated mice. Magnification, x400. Arrows indicate progression of edema and leukocyte presence. Data are presented as the mean \pm standard deviation. ${ }^{*} \mathrm{P}<0.05$ and ${ }^{* *} \mathrm{P}<0.001$ vs. untreated. Ha-P40, Hyaluronic acid-P40; Dex, dexamethasone. 
base cream alone, demonstrating a significant reduction of ear thickness $(\mathrm{P}<0.01$; Fig. 1$)$ and weight $(\mathrm{P}<0.05$; Fig. 2$)$ compared with base cream.

Toxicity. Treatment with the Ha-P40 conjugate cream was well tolerated, judged by the appearance status and the regular body weight increase of the mice (Fig. 3). By contrast, a significant loss of body weight was observed in the group of mice treated for 7 days with dexamethasone compared with untreated mice (Fig. 3; $\mathrm{P}<0.05$ ).

Histological analysis. The aforementioned anti-inflammatory effects were confirmed by histological analysis of the ears. No significant differences were observed between untreated and base cream-treated mice, while a significant reduction in histological score was observed in mice treated with HA-P40 conjugated cream, relative to untreated mice (Fig. 4A; $\mathrm{P}<0.05$ ). Fig. 4B shows a histological image of a healthy mouse without any signs of edema and leucocyte infiltration. Relative to untreated (Fig. 4C) and base cream-treated (Fig. 4D) mice, a marked reductions in edema and leukocyte recruitment were observed in the ears of mice treated with Ha-P40 conjugate (Fig. 4E) and dexamethasone (Fig. 4F) cream.

\section{Discussion}

The humoral defense system of the human body represents the first barrier to external damaging agents including microbial and environmental effects (24). This defense principally constitutes the skin, which acts as a physical barrier (24). When this barrier is crossed, the immune cells intervene on subcutaneous-submucosal level, such as in cases of particularly virulent bacterial or viral infections or in the presence of a skin lesion (24). These cells are primarily macrophages and Epidermal Langerhans Cells, as well as secreted blood factors, including cytokines, complement proteins and interferons (24). The function of the immune cells is to phagocytose and remove pathogens, including bacteria, fungi or viruses (24). This response is mostly enough to eliminate the pathogen but, if the pathogen is particularly virulent, the specific adaptive immunity is activated (24).

However, if the immune system is compromised, due to the prolonged use of antibiotic treatments, tumors or if the pathogenic agent is particularly virulent, the macrophages are not able to respond to pathogenic threats and inflammatory conditions are established (24).

Under normal conditions, macrophages cannot perform appropriately when the invading agent constitutes a large population of microorganisms, or upon invasion by an intercellular-developing microorganism, including a bacterium (Listeria monocytogenes), a fungus (Candida albicans), or a virus, such as the herpes virus, which have an additional immunosuppressive action $(24,25)$.

The immune response is regulated to prevent triggering an inflammatory response, particularly in the barrier tissues such as the skin and mucous membranes of the digestive, respiratory and urinary tracts (24).

On these surfaces, any loss of the delicate physiological balance between the microbiota and the host cells is the basis for a heterogeneous range of mucosal pathologies, such as infective, inflammatory, allergic and vascular disorders in which different pathogenic mechanisms are involved (24). Epidermal macrophages include Langerhans cells, dendritic cells and keratinocytes, which not only present the antigens to the cells of humoral and cell-mediated responses, but are also capable of releasing cytokines to attract lymphocytes towards the site of infection and maintain an inflammatory defense reaction (24).

On the basis of previous data, an effective therapeutic approach for immuno-inflammatory dermatitis should aim to address the altered immune response rather than exert non-specific immunosuppressive effects, which, although effective in the short term, may hinder attempts to achieve physiological homeostasis $(26,27)$.

The use of an immunomodulatory compound, such as Ha-P40 conjugate cream, in skin lesions (for instance, dermatitis) may be able to restore the homeostasis of the dermal immune response. Furthermore, the conjugation of P40 with Ha avoids systemic absorption, thus satisfying the requirement for immune stimulation preparations which, when applied to the skin, are capable of activating a local antibody response and causing infection and inflammation.

In the present study, it was demonstrated that prophylactic treatment with Ha-P40 conjugate cream was able to reduce the signs of inflammation in dermatitis induced by oxazolone. In addition, application of the conjugate cream for seven consecutive days exhibited no local toxic effects. Notably, reduction of ear thickness was similar to that obtained with the positive control drug dexamethasone. Furthermore, conjugation of P40 and Ha appeared to supplement the anti-inflammatory effects observed for base cream containing Ha alone. These clinical features were confirmed by histological analysis of the ears, whereby significant reductions in edema and leukocyte infiltration in mice treated with $\mathrm{Ha} / \mathrm{P} 40$ conjugate cream were observed, relative to untreated mice.

In conclusion, the present results demonstrate that Ha-P40 conjugate cream significantly reduces ear inflammation induced by oxazolone in a murine model of dermatitis, though the underlying mechanisms regarding the effects of Ha-P40 conjugate cream remain unknown. The described effects indicate that the use of this cream may potentially aid to alleviate the symptoms of and/or treat irritant contact dermatitis, triggered by soaps, solvents, chemicals and cosmetics on the skin, or allergic contact dermatitis primarily triggered by metals such as nickel, perfumes, cosmetics, hair dyes and plants, including poison ivy. Further studies are now warranted to elucidate the immunomodulatory mechanisms and full applications of Ha-P40 conjugate cream.

\section{References}

1. Relyveld EH, Bizzini B, Ophir R and Ben-Efraim S: Synergy between low-dose chemotherapy and immunotherapy in mouse L1210 leukemia. Cancer Treat Rep 71: 241-246, 1987.

2. Lallouette P, Bizzini B, Maro B and Schwartz A: Studies on the immunostimulating and anti-tumour activity of a fraction isolated from Corynebacterium granulosum. Dev Biol Stand 38: 111-113, 1977.

3. Henocq E, Bazin JC, Bizzini B and Reynier J: Adjuvant P40 et réactions cutanées à l'antigène. Med Mai infect 8: 415-421, 1978.

4. Bizzini B, Henocq E, Reynier J and Relyveld EH: Experimental and clinical results with the Corynebacterium granulosum-derived immunomodulator P40. Asian Pac J Allergy Immunol 2: 144-153, 1984. 
5. Jacques L, Mathieu D and Bizzini B: Variations in the phagocytic potency of circulating leukocytes from rats which received a thermal injury. Effects of an immunostimulation with Corynebacterium granulosum (author's transl). Pathol Biol (Paris) 26 495-502, 1978 (In French).

6. Mastroeni P, Bizzini B, Bonina L, Iannello D, Merendino RA, Delfino D, Berlinghieri MC,Leonardi MS, Arena A and Liberto MC: The restoration of impaired macrophage functions using an immunomodulator the Corynebacterium granulosum-derived $\mathrm{P} 40$ fraction. Immunopharmacology 10: 27-34, 1985.

7. Bizzini B and Fattal-German M: Potentiation by nonspecific immunostimulation of the efficacy of antibiotics in the treatment of experimental bacterial infections. Biomed Pharmacother 43 753-761, 1989.

8. Fattal-German M and Bizzini B: The Corynebacterium granulosum-derived P40 immune-modulator exerts a synergistic effect on the activity of antiviral drugs in the treatment of experimental viral infections. Biomed Pharmacother 42: 217-220, 1988.

9. Bizzini B, Carlotti M and Fattal-German M: Induction of various cytokines in mice and activation of the complement system in rats as a part of the mechanism of action of the Corynebacterium granulosum-derived P40 immunomodulator. FEMS Microbiol Immunol 5: 171-180, 1992.

10. Mathieu D, Jacques L, Auer J and Bizzini B: E. Coli infections of the lower urinary tract and their treatment by immunomodulation or combined immunomodulation and antigenic therapy. Biomed Pharmacother 42: 271-278, 1988.

11. Henocq E, Bazin JC, Bizzini B and Reynier J: Adjuvant P40 et réactions cutanées à l'antigène. Médecine et Maladies Infectieuses 8: 415-421, 1978.

12. Ickovic MR, Hénocq E, Relyveld EH and Bizzini B: Effect of immunomodulation with the Corynebacterium granulosum-derived Immunomodulator P40 on patients with recurring respiratory infections. J Asthma 21: 29-33, 1984.

13. Henocq E, Veronesi R, Relyveld EH and Bizzini B: Treatment of relapsing chronic infection of the respiratory tract. A comparative study of the effectiveness of non specific immunotherapy with the immunoadjuvant P40 and vaccinotherapy. Rev lnst Med Trap Sao Paulo 26: 105-109, 1984.

14. Henocq E, Arvis G, Delsaux MC and Bizzini B: Traitement des infections urogenitals recidivantes par immunomodulation. Ann Urol (Paris) 19: 371-375, 1985 (In French).

15. Henocq E, Prouvost-Danon A and Bizzini B: Preliminary experimental and clinical results with inactivated allergens conjugated to the Corynebacterium granulosum-derived immunomodulator P40. Boll 1st Sieroter Milan 66: 70-77, 1987.

16. Reynier J, Bizzini B, Bazin JC and Villet R: Immunocompetence, immunostimulation: Experimental facts and clinical perspectives. In: Advances in Immunomodulation. Bizzini B and Bonmassar E (eds). Pythagora Press, Rome-Milan, pp345-362, 1988.
17. Reynier J, Villet R, Bazin JC, Bizzini B, Gandrille C, Guettier C, Henocq E, Hollmann K, Mogenet M, Pompidou A, et al: Immunological investigation and immunotherapy in patients operated on for breast carcinoma. Int Surg 67: 17-24, 1982

18. Esser PR, Wölfle U, Dürr C, von Loewenich FD, Schempp CM, Freudenberg MA, Jakob T and Martin SF: Contact sensitizers induce skin inflammation via ROS production and hyaluronic acid degradation. PLoS One 7: e41340, 2012.

19. Saint-Mezard P, Rosieres A, Krasteva M, Berard F, Dubois B, Kaiserlian D and Nicolas JF: Allergic contact dermatitis. Eur J Dermatol 14: 284-295, 2004.

20. Lehtimäki S, Tillander S, Puustinen A, Matikainen S, Nyman T, Fyhrquist N, Savinko T, Majuri ML, Wolff H, Alenius H and Lauerma A: Absence of CCR4 exacerbates skin inflammation in an oxazolone-induced contact hypersensitivity model. J Invest Dermatol 130: 2743-2751, 2010.

21. Fowler AJ, Sheu MY, Schmuth M, Kao J, Fluhr JW, Rhein L, Collins JL, Willson TM, Mangelsdorf DJ, Elias PM and Feingold KR: Liver X receptor activators display anti-inflammatory activity in irritant and allergic contact dermatitis models: Liver-X-receptor-specific inhibition of inflammation and primary cytokine production. J Invest Dermatol 120: 246-255, 2003.

22. Directive 2010/63/EU of the European parliament and of the council of 22 September 2010 on the protection of animals used for scientific purposes. OJ L 276: 33-79, 2010.

23. Sheu MY, Fowler AJ, Kao J, Schmuth M, Schoonjans K, Auwerx J, Fluhr JW, Man MQ, Elias PM and Feingold KR: Topical peroxisome proliferator activated receptor-alpha activators reduce inflammation in irritant and allergic contact dermatitis models. J Invest Dermatol 118: 94-101, 2002.

24. Skabytska Y, Kaesler S, Volz T and Biedermann T: The role of innate immune signaling in the pathogenesis of atopic dermatitis and consequences for treatments. Semin Immunopathol 38: 29-43, 2016.

25. Kurata S: Fly immunity: Recognition of pathogens and induction of immune responses. Adv Exp Med Biol 708: 205-217, 2010.

26. Celasco G, Moro L, Bozzella R, Mangano K, Quattrocchi C, Aiello C, Donia M, Fagone P and Di Marco R: Efficacy of intracolonic administration of low-molecular-weight heparin CB-01-05, compared to other low-molecular-weight heparins and unfractionated heparin, in experimentally induced colitis in rat. Dig Dis Sci 53: 3170-3175, 2008.

27. Blandino G, Fazio D and Di Marco R: Probiotics: Overview of microbiological and immunological characteristics. Expert Rev Anti Infect Ther 6: 497-508, 2008. 